\title{
Candidate molecular predictors of outcome after aneurysmal subarachnoid haemorrhage: a systematic review of haemoglobin metabolism, inflammation and oxidative injury pathways.
}

\author{
Ben Gaastra ${ }^{1 *}$, Ian Galea ${ }^{2}$ \\ 1. Wessex Neurological Centre, University Hospital Southampton, Southampton, UK. \\ 2. Clinical Neurosciences, Clinical \& Experimental Sciences, Faculty of Medicine, University \\ of Southampton, UK. \\ *email: bgaastra@nhs.net
}

\begin{abstract}
Aneurysmal subarachnoid haemorrhage (aSAH) is a devastating form of stroke associated with significant morbidity and mortality. Very little is known about the predictors of poor outcome and the pathophysiological mechanisms underlying neurological injury following aSAH. Three molecular pathways have been shown to be important: haemoglobin metabolism, inflammation and oxidative injury. The aim of this review is to use a systematic approach to identify a panel of key genes within these three pathways in order to focus future studies investigating predictors of poor outcome and the mechanisms of neurological injury following aSAH. Manual searching and bioinformatic mining tools were used. Studies of experimental or human SAH were included, and outcome was broadly defined to include all encountered readouts such as mortality, neurological scores, and neuropathological markers of tissue damage. If two or more molecules belonged to the same biochemical pathway, this pathway was examined in detail to identify all its components, which were then searched individually for any evidence of association with outcome using the same broad definition as before. This resulted in the identification of 58 candidate genes within the three pathways of interest (haemoglobin metabolism, inflammation and oxidative injury) potentially linked to outcome after aSAH.
\end{abstract}

\section{Introduction}

Aneurysmal subarachnoid haemorrhage (aSAH) results from the release of blood into the subarachnoid space as a consequence of a cerebral artery aneurysm rupture. aSAH is associated with significant morbidity and mortality. Twenty-eight day mortality rates of up to $42 \%$ have been reported ${ }^{1}$ with the largest proportion of patients dying within two days of ictus ${ }^{2}$. Long term morbidity affecting survivors is significant with around half of survivors having cognitive impairments and only a third returning to the same work as prior to haemorrhage ${ }^{3}$. The socioeconomic burden of aSAH in the UK has been estimated as $£ 510$ million annually ${ }^{4}$.

Neurological injury following aSAH can be broadly considered in two categories: firstly, an early brain injury (EBI) occurring within the first 72 hours of ictus, followed by a delayed brain injury occurring days to

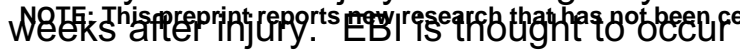

as a consequence of a spike in intracranial pressure and an associated fall in cerebral blood flow. This initiates toxic cascades causing global cerebral ischemia, bloodbrain-barrier (BBB) disruption, cerebral oedema and ultimately cell death ${ }^{5}$. The toxic cascades initiated by EBI and the presence of blood and its breakdown products in the subarachnoid space are thought to cause a delayed brain injury characterised by spreading cortical depression, loss of autoregulation, microthrombi, inflammation, oxidative stress and vasospasm ${ }^{6-8}$.

Blood within the subarachnoid space is broken down and cleared in a multi-step pathway involving a number of scavenging molecules ${ }^{9}$. This clearance system within the central nervous system (CNS) is not as well developed compared to the rest of the body meaning that blood break down products within the CNS persist for a relatively long time causing inflammation

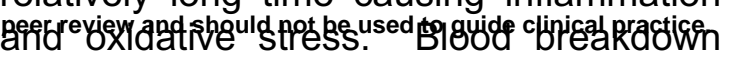


product scavenging molecules have been shown to play an important role in mitigating toxicity and limiting the neurological injury caused by blood breakdown products. Several authoritative reviews ${ }^{9-15}$ have concluded that haemoglobin metabolism, inflammatory and oxidative mechanisms play a significant role in neurological injury following $\mathrm{SAH}$ (Figure 1).

The aim of this review is to identify a panel of molecules within the three themes of haemoglobin metabolism, inflammation and oxidative stress, which are of potential prognostic value after human aSAH. Candidate molecules were identified by an exhaustive search of the literature, using a combination of manual and text-mining methodology.

The candidate molecules identified here will be of use to any investigator who plans to perform a targeted study of the three pathways and outcome after $\mathrm{SAH}$, using genetic, proteomic or metabolomic methods. As an example, in our case, we plan to perform a gene exome sequencing study, during which the exons and upstream regions of the candidates will be sequenced; the association of genetic variation in these regions with clinical outcome after aSAH will be investigated. Further study of these pathways will: (1) provide further evidence of the importance of haemoglobin breakdown product metabolism, inflammation and oxidative stress after human SAH; (2) improve prognostic tools; (3) give insight into the mechanisms of neurological injury following aSAH; (4) identify therapeutic targets for aSAH.

\section{Methods}

\section{$\underline{\text { Rationale }}$}

A combination of manual and semiautomated methodology was used, rather than fully automated methods. In order to illustrate the need for this combined approach, we give an example. In mouse and rat models of $\mathrm{SAH}$, two studies ${ }^{16,17}$ compared RNA expression in cortical tissue from SAH versus control animals, and found that inflammation is a key theme. Blood breakdown products and oxidative stress are intricately linked to inflammation ${ }^{18-23}$, yet these molecules are less likely to feature in automated pathway or network analyses of unbiased proteomic and RNA expression datasets since they are not well described in terms of KEGG pathways and GO ontology terms, and their networks are composed of a small number of molecules. For instance, when the 324 genes identified to be related to $\mathrm{SAH}$ by GLAD4U $^{24}$ (a PubMed gene retrieval and prioritization tool) are run through OverRepresentation Analysis (ORA), Gene Set Enrichment Analysis (GSEA), and Network Topology-based Analysis (NTA) bioinformatic pipelines, oxidative stress and blood breakdown product metabolism are not identified. Hence enrichment of potential candidates within these pathways, based on most likely biologic targets reported in the literature, is needed to study components of these pathways which have been shown to be important in determining outcome after experimental or human SAH.

\section{Overview}

A literature search was performed for molecules shown to be important in influencing outcome after experimental or human aSAH within the three themes of haemoglobin metabolism, inflammation and oxidative stress. Outcome was broadly defined and included all encountered readouts such as mortality, neurological scores, and neuropathological markers of tissue damage.

The search strategy consisted of a manual Pubmed search specifically addressing the three themes of haemoglobin metabolism, inflammation and oxidative stress, supplemented by the full-text literature mining gene retrieval and functional analysis tool SciMiner ${ }^{25}$ (http://hurlab.med.und.edu/SciMiner/).

Publications retrieved were manually screened to ensure that the molecule was positively associated with outcome after $\mathrm{SAH}$. For the reasons explained under "Rationale", a combination of literature and pathway analysis was used in a sequential 
multi-stage process, which is described next.

\section{Description of stages}

The stages of this search are depicted graphically in Figure 2. First, a primary literature search was performed in order to identify molecules (referred to as 'Group A' molecules) relevant to $\mathrm{SAH}$ from the three key themes: blood breakdown product metabolism, inflammation and oxidation. PubMed was searched for articles published in English prior to January 2019 using the following criteria: "oxidation", "anti-oxidant", "oxidative", "redox", "inflammation", "inflammatory", "h(a)emoglobin", "haem/heme" and "iron" in single combination with "subarachnoid h(a)emorrhage". The reference lists of published articles were manually searched for further articles. The results were manually curated to select those molecules related to SAH outcome.

If two or more molecules identified during the primary search belonged to the same biochemical pathway, this pathway was examined using different pathway analysis tools to identify all its components: the Reactome pathway database ${ }^{26}$, KEGG pathway repository ${ }^{27}$, textbooks and the literature. The genes identified by this search that were not present in 'Group A' are referred to as the 'Group B' molecules.

In order to ensure that none of these 'Group B' molecules were overlooked in the primary search, a secondary PubMed search was undertaken. In the secondary search the 'Group B' molecules were individually searched in combination with "subarachnoid h(a)emorrhage". Genes of this molecules were searched using official symbol provided by $\mathrm{HGNC}^{28}$ and any common alternatives. 'Group B' molecules identified in the literature review to have evidence of a role in SAH outcome are referred to as 'Group C' molecules. 'Group D' molecules represent a more comprehensive set of molecules of interest in SAH outcome ('Group A' + 'Group C'). See Figure 2 for a summary of methodology and molecule groups A-D.
To ensure no molecules were missed, Pubmed IDs from both primary and secondary searches were text-mined using the full-text literature mining gene retrieval and functional analysis tool SciMiner ${ }^{25}$ (http://hurlab.med.und.edu/SciMiner/).

Genes which had not been identified manually were traced back to the original publication to identify whether they were associated with outcome after human or experimental $\mathrm{SAH}$.

\section{Results}

As of $3^{\text {rd }}$ August 2019, 1554 publications were retrieved by the primary Pubmed search. Automated text mining of these publications on SciMiner yielded 295 genes. Manual curation of the publications linked to these genes yielded 43 Group $A$ molecules. A further 15 Group $C$ molecules were identified during the secondary Pubmed search (Figure 2).

The final group of molecules $(n=58)$ relevant to outcome after SAH (Group D = $A+C)$ are shown in Table 1. Table 3 provides further detail on the function of each Group D molecule.

\section{Conclusion}

Blood breakdown product metabolism along with inflammatory and oxidative pathways play an important role after $\mathrm{SAH}$. Experimental manipulation and/or genetic variation of molecules in these pathways have been linked to outcome after SAH and they therefore represent a very attractive target for future research studies. Further investigation of these molecules may help inform the mechanisms underlying poor outcome and help develop treatments to mitigate the devastating complications of $\mathrm{SAH}$. It is important to note that these pathways are not independent but intimately linked. Both inflammation and oxidative stress can activate the other pathway, and transcription factors such as Nrf2, PPAR $\gamma$ and NFKB influence both pathways ${ }^{23,29}$. Blood breakdown products can stimulate both inflammation and oxidative stress and their scavengers play an important role to mitigate toxicity by clearing the breakdown products ${ }^{18,22,30-32}$. 
medRxiv preprint doi: https://doi.org/10.1101/19004853; this version posted August 22, 2019. The copyright holder for this preprint (which was not certified by peer review) is the author/funder, who has granted medRxiv a license to display the preprint in perpetuity.

It is made available under a CC-BY-NC-ND 4.0 International license.

In summary, the panel of molecules presented in Table 3 have been identified using a systematic comprehensive search of the literature and will be useful to investigators planning to perform candidate or targeted molecular studies of outcome after SAH. This search will be updated at future intervals. 
Figure 1 - Schematic summarising blood breakdown (1), inflammatory (2) and oxidative (3) pathways involved in SAH.

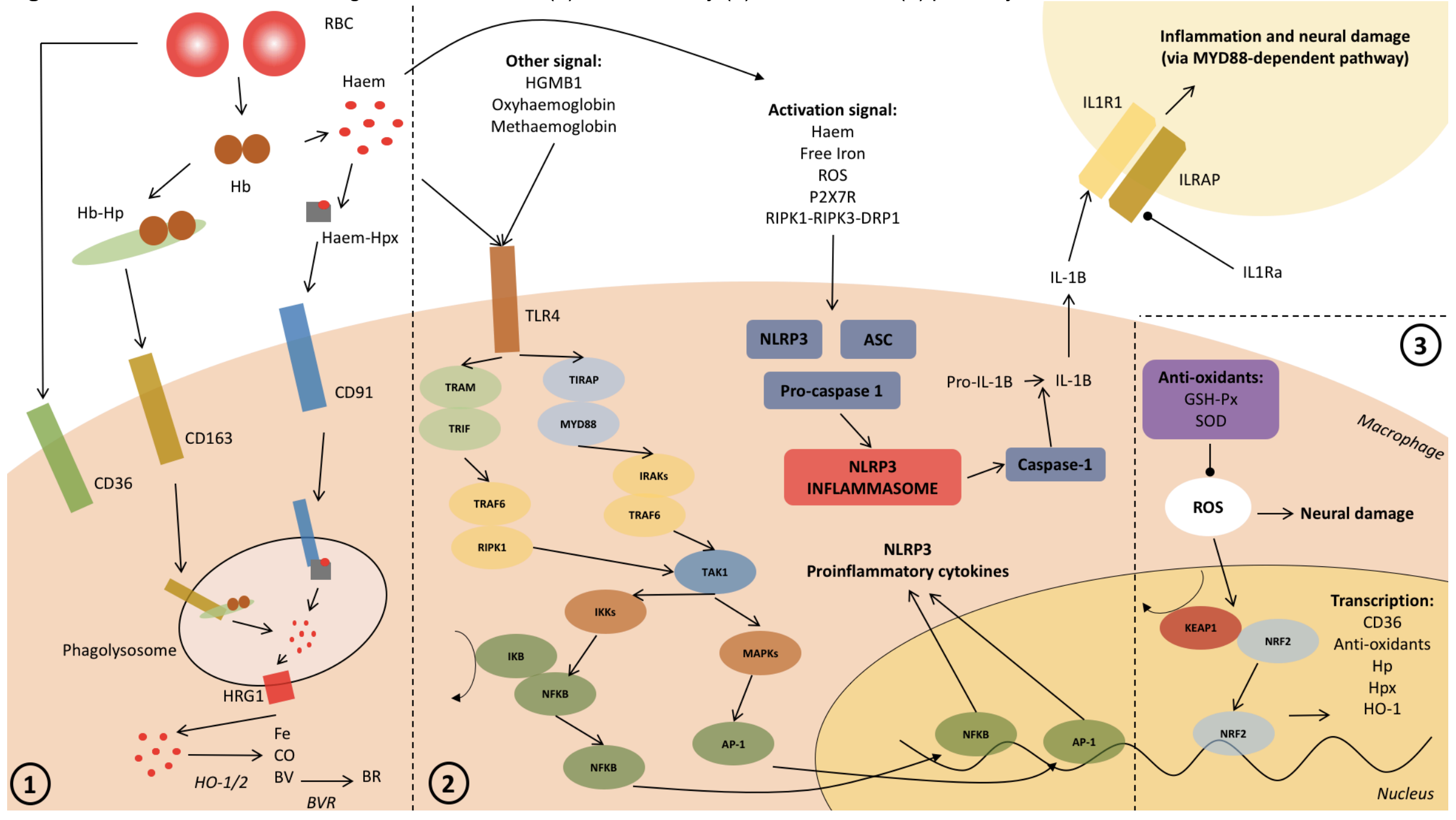


Figure 2 - Methods flowchart

\begin{tabular}{|c|c|c|c|c|}
\hline $\begin{array}{l}\text { STEP } 1 \\
\downarrow\end{array}$ & $\begin{array}{l}\text { Primary Pubmed search by theme } \\
\text { (and outcome after human or } \\
\text { experimental SAH), to identify } \\
\text { Group A molecules (see Table 1) }\end{array}$ & $\begin{array}{l}\text { Theme 1: Blood breakdown } \\
\text { product metabolism }\end{array}$ & Theme 2: Inflammation & Theme 3: Oxidative response \\
\hline $\begin{array}{l}\text { STEP } 2 \\
\downarrow\end{array}$ & $\begin{array}{l}\text { Identification of distinct molecular } \\
\text { pathways within themes } 1-3 \text {, using } \\
\text { multiple biochemical pathway } \\
\text { analyses methodologies (see text) }\end{array}$ & $\begin{array}{l}\text { Blood breakdown } \\
\text { metabolism pathway }\end{array}$ & $\begin{array}{l}\text { Group A molecules belonged } \\
\text { to multiple pathways within the } \\
\text { inflammation theme. However } \\
\text { multiple molecules from TLR4, } \\
\text { NLRP3, and IL1R1 pathways } \\
\text { were identified. }\end{array}$ & $\begin{array}{l}\text { All group A molecules } \\
\text { belonged to one pathway - the } \\
\text { NFE2L2 pathway }\end{array}$ \\
\hline $\mid \begin{array}{l}\text { STEP } 3 \\
\downarrow\end{array}$ & $\begin{array}{l}\text { All known molecules within the } \\
\text { pathways identified in Step } 2- \\
\text { Group Z molecules (see Table 2) }\end{array}$ & $\begin{array}{l}\text { All known molecules within } \\
\text { the blood breakdown } \\
\text { metabolism pathway }\end{array}$ & $\begin{array}{l}\text { All known molecules within the } \\
\text { TLR4, NLRP3, and IL1R1 } \\
\text { pathways }\end{array}$ & $\begin{array}{l}\text { All known molecules within the } \\
\text { NFE2L2 pathway }\end{array}$ \\
\hline $\begin{array}{l}\text { STEP } 4 \\
\downarrow\end{array}$ & \multicolumn{4}{|c|}{ Identification of molecules needing secondary Pubmed search (Group B, where Group B = Group Z - Group A) (see Table 1) } \\
\hline $\begin{array}{l}\text { STEP } 5 \\
\downarrow\end{array}$ & \multicolumn{4}{|c|}{$\begin{array}{l}\text { Secondary Pubmed search to identify 'Group B' molecules with evidence of effect on outcome after human or experimental SAH } \\
\text { (Group C) (see Table 1) }\end{array}$} \\
\hline STEP 6 & \multicolumn{4}{|c|}{$\begin{array}{l}\text { Molecules from primary and secondary Pubmed searches combined (Group D, where Group D = Group A + Group C) (see Table } 1 \\
\text { \& 3) }\end{array}$} \\
\hline
\end{tabular}


medRxiv preprint doi: https://doi.org/10.1101/19004853; this version posted August 22, 2019. The copyright holder for this preprint (which was not certified by peer review) is the author/funder, who has granted medRxiv a license to display the preprint in perpetuity.

It is made available under a CC-BY-NC-ND 4.0 International license .

Table 1. Molecules are denoted by their gene name

\begin{tabular}{|c|c|c|c|c|c|c|}
\hline $\begin{array}{l}\text { Molecule } \\
\text { group }\end{array}$ & $\begin{array}{l}\text { Theme 1: Blood } \\
\text { breakdown product } \\
\text { metabolism }\end{array}$ & \multicolumn{3}{|c|}{ Theme 2: Inflammation } & \multicolumn{2}{|c|}{$\begin{array}{c}\text { Theme 3: Oxidative } \\
\text { response }\end{array}$} \\
\hline A & $\begin{array}{l}\text { HP } \\
\text { HPX } \\
\text { CD163 } \\
\text { ADAM17 } \\
\text { LRP1 } \\
\text { HMOX1 }\end{array}$ & $\begin{array}{l}\text { TLR4 } \\
\text { TLR2 } \\
\text { TIRAP } \\
\text { MYD88 } \\
\text { TICAM1 } \\
\text { RIPK1 } \\
\text { NFKB1 } \\
\text { RELA } \\
\text { NFKBIA } \\
\text { AGER }\end{array}$ & $\begin{array}{l}\text { P2RX7 } \\
\text { PANX1 } \\
\text { NLRP3 } \\
\text { PYCARD } \\
\text { CASP1 } \\
\text { RIPK3 } \\
\text { DNM1L } \\
\text { TNF } \\
\text { IL6 } \\
\text { IL17A }\end{array}$ & $\begin{array}{l}\text { IL23A } \\
\text { IL1B } \\
\text { IL1A } \\
\text { IL1R1 } \\
\text { IL1RN } \\
\text { IL1RM9 } \\
\text { MMAM1 } \\
\text { ICAMPAM1 } \\
\text { VCAMB } \\
\text { HMGG1 }\end{array}$ & $\begin{array}{l}\text { NFE2L2 } \\
\text { KEAP1 } \\
\text { PPARG } \\
\text { BACH1 } \\
\text { CYBB } \\
\text { SOD1 } \\
\text { SOD2 } \\
\text { GPX1 }\end{array}$ & \\
\hline B & $\begin{array}{l}\text { APOL1 } \\
\text { HPR } \\
\text { APOA1 } \\
\text { AMBP } \\
\text { CD36 } \\
\text { BLVRA } \\
\text { FTL } \\
\text { FTH1 } \\
\text { SLC48A1 } \\
\text { HMOX2 }\end{array}$ & $\begin{array}{l}\text { TAB2 } \\
\text { TAB1 } \\
\text { TAB3 } \\
\text { IL12A } \\
\text { IL12B } \\
\text { MAPK1 } \\
\text { MAPK3 } \\
\text { MAPK14 } \\
\text { MAPK11 } \\
\text { MAPK13 } \\
\text { MAPK12 } \\
\text { MAPK8 } \\
\text { MAPK9 } \\
\text { MAPK10 } \\
\text { IRAK4 } \\
\text { IRAK1 }\end{array}$ & \begin{tabular}{|l} 
TRAF6 \\
MAP3K7 \\
JUN \\
FOS \\
CHUK \\
IKBKB \\
IKBKG \\
TRAM1 \\
TXN \\
TXNIP \\
HSP90AB1 \\
SUGT1 \\
RAK3 \\
BTRC \\
FBXW11
\end{tabular} & $\begin{array}{l}\text { NKIRAS1 } \\
\text { NKIRAS2 } \\
\text { PELI1 } \\
\text { TNIP2 } \\
\text { PELI3 } \\
\text { NFKBIB } \\
\text { CUL1 } \\
\text { SQSTM1 } \\
\text { UBE2V1 } \\
\text { MAP2K1 } \\
\text { NFKB2 } \\
\text { S100A12 } \\
\text { SKP1 } \\
\text { UBA52 } \\
\text { RPS27A }\end{array}$ & $\begin{array}{l}\text { CREB1 } \\
\text { NQO1 } \\
\text { GSTO2 } \\
\text { GSTA5 } \\
\text { GSTA1 } \\
\text { GSTA2 } \\
\text { GSTA3 } \\
\text { GSTA4 }\end{array}$ & \begin{tabular}{|l|l} 
GSTM1 & GSTT2 \\
GSTM2 & MGST1 \\
GSTM3 & MGST2 \\
GSTM4 & MGST3 \\
GSTM5 & GSTT2B \\
GSTP1 & GSTO1 \\
GSTT1 & TXNRD1
\end{tabular} \\
\hline C & $\begin{array}{l}\text { FTL } \\
\text { FTH1 }\end{array}$ & $\begin{array}{l}\text { TRAF6 } \\
\text { TAB1 } \\
\text { TAB3 } \\
\text { MAP3K7 }\end{array}$ & $\begin{array}{l}\text { JUN } \\
\text { FOS } \\
\text { CHUK }\end{array}$ & $\begin{array}{l}\text { IKBKB } \\
\text { TXN } \\
\text { TXNIP }\end{array}$ & $\begin{array}{l}\text { CREB1 } \\
\text { NQO1 } \\
\text { GSTA1 }\end{array}$ & \\
\hline D & $\begin{array}{l}\text { HP } \\
\text { HPX } \\
\text { CD163 } \\
\text { ADAM17 } \\
\text { LRP1 } \\
\text { HMOX1 } \\
\text { FTL } \\
\text { FTH1 }\end{array}$ & $\begin{array}{l}\text { TLR4 } \\
\text { TLR2 } \\
\text { TIRAP } \\
\text { MYD88 } \\
\text { TICAM1 } \\
\text { RIPK1 } \\
\text { RIPK3 } \\
\text { DNM1L } \\
\text { NFKB1 } \\
\text { RELA } \\
\text { NFKBIA } \\
\text { AGER } \\
\text { P2RX7 } \\
\text { PANX1 }\end{array}$ & $\begin{array}{l}\text { NLRP3 } \\
\text { PYCARD } \\
\text { CASP1 } \\
\text { TNF } \\
\text { IL6 } \\
\text { IL17A } \\
\text { IL23A } \\
\text { IL1B } \\
\text { IL1A } \\
\text { IL1R1 } \\
\text { IL1RN } \\
\text { ILMP9 } \\
\text { ICAM1 } \\
\text { ICAM1 }\end{array}$ & $\begin{array}{l}\text { HMGB1 } \\
\text { TRAF6 } \\
\text { TAB1 } \\
\text { MAP3K7 } \\
\text { JUN } \\
\text { FOS } \\
\text { CHUK } \\
\text { IKBKB } \\
\text { TXN } \\
\text { TXNIP } \\
\text { S100B }\end{array}$ & $\begin{array}{l}\text { NFE2L2 } \\
\text { KEAP1 } \\
\text { PPARG } \\
\text { BACH1 } \\
\text { CYBB } \\
\text { SOD1 } \\
\text { SOD2 } \\
\text { GPX1 } \\
\text { CREB1 } \\
\text { NQO1 } \\
\text { GSTA1 }\end{array}$ & \\
\hline
\end{tabular}


Table 2. Molecules are denoted by their gene name

\begin{tabular}{|c|c|c|c|c|c|c|c|c|c|}
\hline Theme & \multirow{2}{*}{\multicolumn{2}{|c|}{$\begin{array}{l}\text { 1: Blood breakdown } \\
\text { product metabolism } \\
\text { Scavenging of heme }\end{array}$}} & \multicolumn{6}{|c|}{ 2: Inflammation } & \multirow{2}{*}{$\begin{array}{l}\text { 3: Oxidative response } \\
\text { NFE2L2 } \\
\end{array}$} \\
\hline Pathway of interest & & & TLR4 & & IL1R1 & & & NLRP3 & \\
\hline Source of pathway & Reactom & & KEGG & & KEGG \& Re & ctome & & Reactome & KEGG \\
\hline Pathway Identifier & $\begin{array}{l}\text { Scaveng } \\
\text { plasma }\end{array}$ & ff heme in & $\begin{array}{l}\text { N00186 } \\
\text { N00188 }\end{array}$ & & $\begin{array}{l}\text { N00435 } \\
\text { N00438 } \\
\text { IL-1 family s }\end{array}$ & naling & & NLR signalling pathways & N00243 \\
\hline Components & $\begin{array}{l}\text { HPX } \\
\text { LRP1 } \\
\text { APOL1 } \\
\text { HPR } \\
\text { APOA1 } \\
\text { HP } \\
\text { CD163 } \\
\text { AMBP }\end{array}$ & & $\begin{array}{l}\text { TLR4 } \\
\text { TIRAP } \\
\text { MYD88 } \\
\text { IRAK4 } \\
\text { IRAK1 } \\
\text { TRAF6 } \\
\text { MAP3K7 } \\
\text { TAB2 } \\
\text { CHUK } \\
\text { IKBKB } \\
\text { IKBKG } \\
\text { NFKBIA } \\
\text { NFKB1 } \\
\text { RELA } \\
\text { TNF }\end{array}$ & $\begin{array}{l}\text { IL6 } \\
\text { IL12A } \\
\text { IL12B } \\
\text { MAPK1 } \\
\text { MAPK3 } \\
\text { MAPK14 } \\
\text { MAPK11 } 11 \\
\text { MAPK13 } 13 \\
\text { MAPK12 } 2 \\
\text { MAPK8 } \\
\text { MAPK9 } \\
\text { MAPK10 } \\
\text { FOS } \\
\text { JUN }\end{array}$ & $\begin{array}{l}\text { IL1A } \\
\text { IL1B } \\
\text { IL1R1 } \\
\text { IL1RAP } \\
\text { MYD88 } \\
\text { IRAK1 } \\
\text { IRAK4 } \\
\text { TRAF6 } \\
\text { TAB1 } \\
\text { TAB2 } \\
\text { TAB3 } \\
\text { MAP3K7 } \\
\text { MAP2K3 } \\
\text { MAP2K6 } \\
\text { MAPK14 } \\
\text { MAPKAPK2 } \\
\text { ZFP36 } \\
\text { MAP2K4 } \\
\text { MAP2K7 } \\
\text { MAPK8 } \\
\text { MAPK9 } \\
\text { MAPK10 } \\
\text { FOS } \\
\text { JUN } \\
\text { TLR2 }\end{array}$ & $\begin{array}{l}\text { TLR4 } \\
\text { TIRAP } \\
\text { CHUK } \\
\text { IKBKB } \\
\text { IKBKG } \\
\text { NFKBIA } \\
\text { NFKB1 } \\
\text { RELA } \\
\text { TNF } \\
\text { IL6 } \\
\text { IL12A } \\
\text { IL12B } \\
\text { MAPK1 } \\
\text { MAPK3 } \\
\text { MAPK14 } \\
\text { MAPK11 } \\
\text { MAPK13 } \\
\text { MAPK12 } \\
\text { MAPK10 } \\
\text { IRAK3 } \\
\text { BTRC } \\
\text { FBXW11 } \\
\text { NKIRAS1 } \\
\text { NKIRAS2 } \\
\text { PELI1 }\end{array}$ & $\begin{array}{l}\text { TNIP2 } \\
\text { PELI3 } \\
\text { NFKBIB } \\
\text { AGER } \\
\text { CUL1 } \\
\text { SQSTM1 } \\
\text { UBE2V1 } \\
\text { MAP2K1 } \\
\text { NFKB2 } \\
\text { S100A12 } \\
\text { SKP1 } \\
\text { UBA52 } \\
\text { RPS27A } \\
\text { RBX1 } \\
\text { UBE2N } \\
\text { MAP3K8 } \\
\text { IL1R2 } \\
\text { IL1RN } \\
\text { SAA1 } \\
\text { UBC } \\
\text { UBB } \\
\text { HMGB1 } \\
\text { APP } \\
\text { S100B } \\
\text { MAP3K7 }\end{array}$ & $\begin{array}{l}\text { TXN } \\
\text { TXNIP } \\
\text { HSP90AB1 } \\
\text { SUGT1 } \\
\text { NLRP3 } \\
\text { PYCARD } \\
\text { CASP1 }\end{array}$ & $\begin{array}{l}\text { KEAP1 } \\
\text { NFE2L2 } \\
\text { HMOX1 } \\
\text { NQO1 } \\
\text { GSTO2 } \\
\text { GSTA5 } \\
\text { GSTA1 } \\
\text { GSTA2 } \\
\text { GSTA3 } \\
\text { GSTA4 } \\
\text { GSTM1 } \\
\text { GSTM2 } \\
\text { GSTM3 } \\
\text { GSTM4 } \\
\text { GSTM5 } \\
\text { GSTP1 } \\
\text { GSTT1 } \\
\text { GSTT2 } \\
\text { MGST1 } \\
\text { MGST2 } \\
\text { MGST3 } \\
\text { GSTT2B } \\
\text { GSTO1 } \\
\text { TXNRD1 }\end{array}$ \\
\hline $\begin{array}{l}\text { Molecules identified } \\
\text { by additional } \\
\text { literature review }\end{array}$ & $\begin{array}{l}\text { CD36 } \\
\text { BLVRA } \\
\text { FTL }\end{array}$ & $\begin{array}{l}\text { FTH1 } \\
\text { SLC48A1 } \\
\text { HMOX2 }\end{array}$ & TRAM1 & & & & & & CREB1 \\
\hline
\end{tabular}


medRxiv preprint doi: https://doi.org/10.1101/19004853; this version posted August 22, 2019. The copyright holder for this preprint (which was not certified by peer review) is the author/funder, who has granted medRxiv a license to display the preprint in perpetuity.

Table 3. Molecules are denoted by their gene name

\begin{tabular}{|c|c|c|c|}
\hline & Gene name & Symbol (HGNC) & Function \\
\hline \multirow{39}{*}{$\begin{array}{l}\text { Inflammatory } \\
\text { pathway } \\
\text { molecules }\end{array}$} & Purinergic receptor P2X 7 & $\mathrm{P} 2 \mathrm{R} \times 7$ & ATP receptor \\
\hline & Pannexin-1 & PANX1 & Gap junction protein \\
\hline & $\begin{array}{l}\text { Nuclear factor kappa B } \\
\text { subunit } 1\end{array}$ & NFKB1 & $\begin{array}{l}\text { Component of NFkB transcription } \\
\text { factor }\end{array}$ \\
\hline & RELA proto-oncogene & RELA & $\begin{array}{l}\text { Component of NFkB transcription } \\
\text { factor }\end{array}$ \\
\hline & NKKB inhibitor alpha & NFKBIA & $\mathrm{I \kappa B}$ \\
\hline & $\begin{array}{l}\text { NLR family pyrin domain } \\
\text { containing } 3\end{array}$ & NLRP3 & $\begin{array}{l}\text { Inflammasome complex } \\
\text { component }\end{array}$ \\
\hline & $\begin{array}{l}\text { PYD and CARD domain } \\
\text { containing }\end{array}$ & PYCARD & $\begin{array}{l}\text { Inflammasome complex } \\
\text { component }\end{array}$ \\
\hline & Caspase 1 & CASP1 & $\begin{array}{l}\text { Inflammasome complex } \\
\text { component }\end{array}$ \\
\hline & $\begin{array}{l}\text { Receptor interacting } \\
\text { serine/threonine kinase } 1\end{array}$ & RIPK1 & Inflammasome complex activator \\
\hline & $\begin{array}{l}\text { Receptor interacting } \\
\text { serine/threonine kinase } 3\end{array}$ & RIPK3 & Inflammasome complex activator \\
\hline & Dynamin 1 like & DNM1L & Inflammasome complex activator \\
\hline & TNF $\alpha$ & TNF & Cytokine \\
\hline & Interleukin 6 & IL6 & Cytokine \\
\hline & Interleukin 17 & IL17A & Cytokine \\
\hline & Interleukin 23 & IL23A & Cytokine \\
\hline & Interleukin $1 \beta$ & IL1B & Cytokine \\
\hline & Interleukin $1 \alpha$ & IL1A & Cytokine \\
\hline & Interleukin 1 receptor type 1 & IL1R1 & Interleukin 1 receptor \\
\hline & Toll-like receptor 4 & TLR4 & Pattern recognition receptor \\
\hline & Toll-like receptor 2 & TLR2 & Pattern recognition receptor \\
\hline & $\begin{array}{l}\text { TIR domain containing } \\
\text { adaptor protein }\end{array}$ & TIRAP & $\begin{array}{l}\text { Adaptor protein in IL-1 and TLR } \\
\text { signalling pathways }\end{array}$ \\
\hline & $\begin{array}{l}\text { Mitogen-activated protein } \\
\text { kinase kinase } 7\end{array}$ & MAP3K7 & $\begin{array}{l}\text { Adaptor protein in IL-1 and TLR } \\
\text { signalling pathways }\end{array}$ \\
\hline & $\begin{array}{l}\text { TGF-beta activated kinase } 1 \\
\text { (MAP3K7) binding protein } 1\end{array}$ & TAB1 & MAP3K7 modifier \\
\hline & $\begin{array}{l}\text { TNF receptor associated } \\
\text { factor } 6\end{array}$ & TRAF6 & $\begin{array}{l}\text { Adaptor protein in IL-1 and TLR } \\
\text { signalling pathways }\end{array}$ \\
\hline & Jun proto-oncogene & JUN & AP-1 component \\
\hline & Fos proto-oncogene & FOS & AP-1 component \\
\hline & $\begin{array}{l}\text { Conserved helix-loop-helix } \\
\text { ubiquitous kinase }\end{array}$ & $\mathrm{CHUK}$ & IKK complex component \\
\hline & $\begin{array}{l}\text { Inhibitor of nuclear factor } \\
\text { kappa B kinase subunit beta }\end{array}$ & IKBKB & IKK complex component \\
\hline & $\begin{array}{l}\text { Myeloid differentiation primary } \\
\text { response } 88\end{array}$ & MYD88 & $\begin{array}{l}\text { Adaptor protein in IL-1 and TLR } \\
\text { signalling pathways }\end{array}$ \\
\hline & $\begin{array}{l}\text { Toll like receptor adaptor } \\
\text { molecule } 1\end{array}$ & TICAM1 & $\begin{array}{l}\text { Adaptor protein in TLR signalling } \\
\text { pathways }\end{array}$ \\
\hline & High mobility group box 1 & HMGB1 & TLR4 activator \\
\hline & $\begin{array}{l}\text { Advanced glycosylation end- } \\
\text { product specific receptor }\end{array}$ & AGER & HMGB1 receptor \\
\hline & $\begin{array}{l}\text { Interleukin } 1 \text { receptor } \\
\text { antagonist }\end{array}$ & IL1RN & Inhibitor of IL1 at IL1R1 \\
\hline & Matrix metallopeptidase 9 & MMP9 & Matrix metalloproteinase \\
\hline & $\begin{array}{l}\text { Intercellular adhesion } \\
\text { molecule } 1\end{array}$ & ICAM1 & Cell surface glycoprotein \\
\hline & $\begin{array}{l}\text { Vascular cell adhesion } \\
\text { molecule } 1\end{array}$ & VCAM1 & Cell adhesion molecule \\
\hline & Thioredoxin & TXN & Antioxidant \\
\hline & $\begin{array}{l}\text { Thioredoxin-interacting } \\
\text { protein }\end{array}$ & TXNIP & TXN modifier \\
\hline & $\begin{array}{l}\text { S100 calcium binding protein } \\
\text { B }\end{array}$ & S100B & $\begin{array}{l}\text { Intracellular calcium binding } \\
\text { protein }\end{array}$ \\
\hline
\end{tabular}


medRxiv preprint doi: https://doi.org/10.1101/19004853; this version posted August 22, 2019. The copyright holder for this preprint (which was not certified by peer review) is the author/funder, who has granted medRxiv a license to display the preprint in perpetuity.

It is made available under a CC-BY-NC-ND 4.0 International license.

\begin{tabular}{|c|c|c|c|}
\hline \multirow[t]{11}{*}{$\begin{array}{l}\text { Oxidative } \\
\text { pathway } \\
\text { molecules }\end{array}$} & $\begin{array}{l}\text { Nuclear factor erythroid } 2 \text { like } \\
2\end{array}$ & NFE2L2 & $\begin{array}{l}\text { Transcription factor promoting } \\
\text { expression of anti-inflammatory } \\
\text { genes }\end{array}$ \\
\hline & $\begin{array}{l}\text { Kelch like ECH associated } \\
\text { protein } 1\end{array}$ & KEAP1 & Regulator of NFE2L2 activity \\
\hline & $\begin{array}{l}\text { Peroxisome proliferator } \\
\text { activated receptor gamma }\end{array}$ & PPARG & Nuclear receptor \\
\hline & $\begin{array}{l}\text { BTB domain and CNC } \\
\text { homolog } 1\end{array}$ & $\mathrm{BACH} 1$ & Regulator of NFE2L2 activity \\
\hline & $\begin{array}{l}\text { cAMP responsive element } \\
\text { binding protein } 1\end{array}$ & CREB1 & $\begin{array}{l}\text { Regulator of NFE2L2 and NFKB } \\
\text { activity }\end{array}$ \\
\hline & Cytochrome b-245 beta chain & CYBB & NADPH oxidase \\
\hline & Superoxide dismutase 1 & SOD1 & Antioxidant \\
\hline & Superoxide dismutase 2 & SOD2 & Antioxidant \\
\hline & Glutathione peroxidase 1 & GPX1 & Antioxidant \\
\hline & $\begin{array}{l}\text { NAD(P)H quinone } \\
\text { oxidoreductase }\end{array}$ & NQO1 & Antioxidant \\
\hline & glutathione S-transferase- $\alpha 1$ & GSTA1 & Antioxidant \\
\hline \multirow{8}{*}{$\begin{array}{l}\text { Blood } \\
\text { breakdown } \\
\text { product } \\
\text { scavenger } \\
\text { molecules }\end{array}$} & Haptoglobin & $\mathrm{HP}$ & Haemoglobin scavenger \\
\hline & Hemepexin & HPX & Heme scavenger \\
\hline & CD163 & CD163 & $\begin{array}{l}\text { Facilitates endocytosis of } \\
\text { haemoglobin-haptoglobin } \\
\text { complexes into } \\
\text { microglia/macrophages }\end{array}$ \\
\hline & LDL receptor related protein 1 & LRP1 & $\begin{array}{l}\text { Facilitates endocytosis of heme- } \\
\text { hemopexin complexes }\end{array}$ \\
\hline & $\begin{array}{l}\text { ADAM metallopeptidase } \\
\text { domain } 17\end{array}$ & ADAM17 & $\begin{array}{l}\text { Facilitates shedding of CD163 } \\
\text { from cell surface }\end{array}$ \\
\hline & Ferritin light chain & FTL & Intracellular iron storage protein \\
\hline & Ferritin heavy chain 1 & FTH1 & Intracellular iron storage protein \\
\hline & Heme oxygenase 1 & HMOX1 & Cleaves heme forming biliverdin \\
\hline
\end{tabular}




\section{References}

1 Ingall, T., Asplund, K., Mähönen, M. \& Bonita, R. A multinational comparison of subarachnoid hemorrhage epidemiology in the WHO MONICA stroke study. Stroke 31, 1054-1061 (2000).

2 Broderick, J. P., Brott, T. G., Duldner, J. E., Tomsick, T. \& Leach, A. Initial and recurrent bleeding are the major causes of death following subarachnoid hemorrhage. Stroke 25, 1342-1347 (1994).

3 Rinkel, G. J. \& Algra, A. Long-term outcomes of patients with aneurysmal subarachnoid haemorrhage. Lancet Neurol 10, 349-356, doi:10.1016/S14744422(11)70017-5 (2011).

4 Rivero-Arias, O., Gray, A. \& Wolstenholme, J. Burden of disease and costs of aneurysmal subarachnoid haemorrhage (aSAH) in the United Kingdom. Cost Eff Resour Alloc 8, 6, doi:10.1186/1478-7547-8-6 (2010).

5 Cahill, J., Cahill, W. J., Calvert, J. W., Calvert, J. H. \& Zhang, J. H. Mechanisms of early brain injury after subarachnoid hemorrhage. J Cereb Blood Flow Metab 26, 13411353, doi:10.1038/sj.jcbfm.9600283 (2006).

6 D'Souza, S. Aneurysmal Subarachnoid Hemorrhage. J Neurosurg Anesthesiol 27, 222240, doi:10.1097/ANA.0000000000000130 (2015).

7 Macdonald, R. L., Pluta, R. M. \& Zhang, J. H. Cerebral vasospasm after subarachnoid hemorrhage: the emerging revolution. Nat Clin Pract Neurol 3, 256-263, doi:10.1038/ncpneuro0490 (2007).

8 Gaetani, P., Pasqualin, A., Rodriguez y Baena, R., Borasio, E. \& Marzatico, F. Oxidative stress in the human brain after subarachnoid hemorrhage. J Neurosurg 89, 748-754, doi:10.3171/jns.1998.89.5.0748 (1998).

9 Bulters, D. et al. Haemoglobin scavenging in intracranial bleeding: biology and clinical implications. Nat Rev Neurol 14, 416-432, doi:10.1038/s41582-018-0020-0 (2018).

10 Zheng, V. Z. \& Wong, G. K. C. Neuroinflammation responses after subarachnoid hemorrhage: A review. J Clin Neurosci 42, 7-11, doi:10.1016/j.jocn.2017.02.001 (2017).

11 Ayer, R. E. \& Zhang, J. H. Oxidative stress in subarachnoid haemorrhage: significance in acute brain injury and vasospasm. Acta Neurochir Suppl 104, 33-41 (2008).

12 Miller, B. A., Turan, N., Chau, M. \& Pradilla, G. Inflammation, vasospasm, and brain injury after subarachnoid hemorrhage. Biomed Res Int 2014, 384342, doi:10.1155/2014/384342 (2014).

13 Ciurea, A. V., Palade, C., Voinescu, D. \& Nica, D. A. Subarachnoid hemorrhage and cerebral vasospasm - literature review. J Med Life 6, 120-125 (2013).

14 Macdonald, R. L. \& Schweizer, T. A. Spontaneous subarachnoid haemorrhage. Lancet 389, 655-666, doi:10.1016/S0140-6736(16)30668-7 (2017).

15 Yang, Y., Chen, S. \& Zhang, J. M. The Updated Role of Oxidative Stress in Subarachnoid Hemorrhage. Curr Drug Deliv 14, 832-842, doi:10.2174/1567201813666161025115531 (2017).

16 Peng, J. et al. High-Throughput Sequencing and Co-Expression Network Analysis of IncRNAs and mRNAs in Early Brain Injury Following Experimental Subarachnoid Haemorrhage. Sci Rep 7, 46577, doi:10.1038/srep46577 (2017). 
17 Zheng, B. et al. Expression signatures of long non-coding RNAs in early brain injury following experimental subarachnoid hemorrhage. Mol Med Rep 12, 967-973, doi:10.3892/mmr.2015.3474 (2015).

18 Dutra, F. F. \& Bozza, M. T. Heme on innate immunity and inflammation. Front Pharmacol 5, 115, doi:10.3389/fphar.2014.00115 (2014).

$19 \mathrm{Wu}, \mathrm{Y}$. et al. Peroxisome proliferator-activated receptor gamma agonist rosiglitazone attenuates oxyhemoglobin-induced Toll-like receptor 4 expression in vascular smooth muscle cells. Brain Res 1322, 102-108, doi:10.1016/j.brainres.2010.01.073 (2010).

20 Kwon, M. S. et al. Methemoglobin is an endogenous toll-like receptor 4 ligandrelevance to subarachnoid hemorrhage. Int J Mol Sci 16, 5028-5046, doi:10.3390/ijms16035028 (2015).

21 Dutra, F. F. et al. Hemolysis-induced lethality involves inflammasome activation by heme. Proc Natl Acad Sci U S A 111, E4110-4118, doi:10.1073/pnas.1405023111 (2014).

22 Nakamura, K. et al. Activation of the NLRP3 inflammasome by cellular labile iron. Exp Hematol 44, 116-124, doi:10.1016/j.exphem.2015.11.002 (2016).

23 Liu, G. H., Qu, J. \& Shen, X. NF-kappaB/p65 antagonizes Nrf2-ARE pathway by depriving CBP from Nrf2 and facilitating recruitment of HDAC3 to MafK. Biochim Biophys Acta 1783, 713-727, doi:10.1016/j.bbamcr.2008.01.002 (2008).

24 Jourquin, J., Duncan, D., Shi, Z. \& Zhang, B. GLAD4U: deriving and prioritizing gene lists from PubMed literature. BMC Genomics 13 Suppl 8, S20, doi:10.1186/14712164-13-S8-S20 (2012).

25 Hur, J., Schuyler, A. D., States, D. J. \& Feldman, E. L. SciMiner: web-based literature mining tool for target identification and functional enrichment analysis.

Bioinformatics 25, 838-840, doi:10.1093/bioinformatics/btp049 (2009).

26 Fabregat, A. et al. The Reactome Pathway Knowledgebase. Nucleic Acids Res 46, D649-D655, doi:10.1093/nar/gkx1132 (2018).

27 Kanehisa, M., Sato, Y., Kawashima, M., Furumichi, M. \& Tanabe, M. KEGG as a reference resource for gene and protein annotation. Nucleic Acids Res 44, D457-462, doi:10.1093/nar/gkv1070 (2016).

28 Braschi, B. et al. Genenames.org: the HGNC and VGNC resources in 2019. Nucleic Acids Res 47, D786-D792, doi:10.1093/nar/gky930 (2019).

29 Zhao, X. R., Gonzales, N. \& Aronowski, J. Pleiotropic role of PPARy in intracerebral hemorrhage: an intricate system involving Nrf2, RXR, and NF-KB. CNS Neurosci Ther 21, 357-366, doi:10.1111/cns.12350 (2015).

30 Reeder, B. J. et al. Toxicity of myoglobin and haemoglobin: oxidative stress in patients with rhabdomyolysis and subarachnoid haemorrhage. Biochem Soc Trans 30, 745-748, doi:10.1042/ (2002).

31 Macdonald, R. L. \& Weir, B. K. A review of hemoglobin and the pathogenesis of cerebral vasospasm. Stroke 22, 971-982 (1991).

32 Winterbourn, C. C. Free-radical production and oxidative reactions of hemoglobin. Environ Health Perspect 64, 321-330, doi:10.1289/ehp.8564321 (1985). 